\title{
Sublingual immunotherapy for allergic rhinitis: subjective versus objective tools to evaluate its success*
}

\author{
Daiju Sakurai', Syuji Yonekura' ${ }^{1}$ Tomohisa linuma ${ }^{1,2}$, Toshioki Sakurai', \\ Yuki Morimoto', Yukiyoshi Mita', Tomoyuki Arai', Satoshi Suzuki', \\ Yusuke Okuma', Shinya Kaneko³, Yoshitaka Okamoto' \\ ' Department of Otolaryngology, Head and Neck Surgery, Graduate School of Medicine, Chiba University, Chiba, Japan \\ ${ }^{2}$ Department of Advanced Allergology of the Airway, Graduate School of Medicine, Chiba University, Chiba, Japan \\ ${ }^{3}$ Torii Pharmaceutical Co., Ltd., Tokyo, Japan
}

Rhinology 54: 221-230, 2016

DOI:10.4193/Rhino15.223

*Received for publication:

July 31, 2015

Accepted: February 11, 2016

\begin{abstract}
Background: Biomarkers that enable objective evaluation of the clinical effects of immunotherapy for allergic rhinitis have yet to be identified.

Methods: This study included 40 patients who were enrolled in a large randomized, double-blind, placebo-controlled, multicenter study examining the efficacy of sublingual immunotherapy (SLIT) using Japanese cedar (JC) pollen extract during two consecutive pollen seasons from 2010 to 2012. Based on changes in total nasal symptom medication score, patients in the SLIT and placebo groups were subdivided into two subgroups: good responders and poor responders. The levels of JC pollen-specific IL-10+Foxp3+ cells and specific Th2 cytokine-producing cells were measured and the association with the efficacy of SLIT was analysed.
\end{abstract}

Results: The total nasal symptom medication score was significantly lower in the SLIT group compared with the placebo group. The number of JC pollen-specific Th2 cytokine-producing cells increased during the pollen season in the placebo group and in poor responders in the SLIT group; however, the increases were inhibited in the good responders in the SLIT group. The number of JC pollen-specific IL-10+Foxp3+ cells increased only in these good responders.

Conclusions: Changes in levels of allergen-specific Th2 cytokine-producing cells and IL-10+Foxp3+ cells could be objective biomarkers for SLIT.

Key words: allergic rhinitis, biological markers, sublingual immunotherapy, pollinosis

\section{Introduction}

A number of clinical trials of sublingual immunotherapy (SLIT) for allergic rhinitis (AR) have been conducted, and recent meta-analyses have shown significant improvements in the SLIT group compared with the placebo group ${ }^{(1-4)}$. Japanese cedar (JC) (Cryptomeria japonica) pollinosis is the most common type of seasonal AR in Japan. Previous randomized, double-blind, placebo-controlled studies of SLIT for JC pollinosis demonstrated that nasal symptoms and quality of life (QOL) were significantly improved ${ }^{(5-8)}$. Based on these studies, a phase III clinical trial of SLIT for JC pollinosis was conducted from 2010 to 2012, and this study demonstrated the efficacy and safety of SLIT ${ }^{(9)}$.

However, because nasal symptoms were influenced by the amount of airborne pollen and the duration of dispersal in each year it is possible that placebo effects may have influenced the results. In our previous clinical trials and in the phase III trial of SLIT for JC pollinosis, the mean total nasal symptom-medication score (TNSMS) was significantly lower in the SLIT group than in the placebo group during the pollen season; however, there were patients whose symptoms were not improved in the SLIT group. In the phase-III trial, 39\% of patients in the SLIT group had moderate symptoms or more than moderate symptoms in the second pollen season. Conversely, there was a relatively large number of patients with mild symptoms or with unimpaired QOL in the placebo group ${ }^{(6,7,9)}$. It is therefore difficult to determine the therapeutic efficacy of SLIT at the individual 
patient level by the nasal symptom or QOL scores.

To evaluate the efficacy of SLIT in individual patients in an objective fashion it is necessary to identify a biomarker reflecting the therapeutic efficacy, and to establish this as an assessment method. It has been reported there were several markers associated with the mechanism of SLIT ${ }^{(10-12)}$, but an effective evaluation method using these markers has not been established. We have evaluated biomarkers reflecting positive effects of SLIT in the clinical trials and have reported that levels of JC pollen-specific interleukin (IL)-10-producing Foxp $3^{+}$regulatory $\mathrm{T}$ (Treg) cells and specific Th2 cells could be involved in the effect of SLIT ${ }^{(5-7)}$. This study included patients $(n=40)$ recruited at our institute who were involved in the phase III trial. Patients in the SLIT and placebo groups were subdivided into two subgroups, good responders and poor responders, based on the change in TNSMS for two consecutive pollen seasons. The changes in total immunoglobulin $\mathrm{E}(\mathrm{lg} \mathrm{E}), \mathrm{JC}$ pollen-specific $\lg \mathrm{E}$, immunoglobulin G4 (IgG4), specific IL-10+Foxp3+ Treg cells, and specific Th2 cytokine-producing cells were examined in the subgroups, and the associations with the efficacy of SLIT were analysed.

\section{Materials and methods}

\section{Patients}

A large randomized, double-blind, multicenter, comparative study examining the efficacy of SLIT using the JC pollen extract was conducted in Japan over two consecutive JC pollen seasons from October 2010 to April 2012; a total of 481 patients completed the study ${ }^{(9)}$. The current study included 40 patients from this clinical trial who had been allocated and recruited at Chiba University Hospital and cooperating medical institutions (Chiba Kaihin Municipal Hospital, Nakano ENT Clinic). These patients met the inclusion criteria of age 12-64 years, JC pollen-specific IgE levels (ImmunoCAP) of class 3 or higher, and symptoms of $J C$ pollinosis for the previous 2 years, and did not meet any of the exclusion criteria of perennial AR, drug-induced rhinitis, nonallergic rhinitis requiring treatment, previous immunotherapy for JC pollinosis, immunotherapy within the previous 5 years, an asthma attack within the previous 5 years, and pregnancy. Patients were randomized into a JC pollen extract (SLIT) group and a placebo group in a 1:1 allocation ratio (Table 1).

The protocol was approved by the Ethics Committee of Chiba University, and written informed consent was obtained from each patient prior to participation in the study. The study was conducted in accordance with the Declaration of Helsinki and Good Clinical Practice guidelines.

\section{Study protocols}

The patients in the SLIT group received standardized JC pollen extract (Torii Pharmaceutical Co. Ltd., Tokyo, Japan), and the patients in the placebo group received an inactive placebo. Patients were instructed to place the study drug under their
Table 1. Demographics and baseline characteristics of patients.

\begin{tabular}{|c|c|c|c|c|}
\hline & & $\begin{array}{l}\text { JC pollen } \\
\text { extract }\end{array}$ & Placebo & P-value \\
\hline Number & & 20 & 20 & \\
\hline $\operatorname{Sex}(M / F)$ & & $3 / 17$ & $2 / 18$ & \\
\hline \multirow[t]{2}{*}{ Age (years) } & Mean $\pm S D$ & $43.7 \pm 8.1$ & $44.3 \pm 9.0$ & n.s. ${ }^{\#}$ \\
\hline & Range & $29-61$ & $31-60$ & \\
\hline \multirow[t]{2}{*}{ Total lgE } & Mean $\pm S D$ & $100.8 \pm 101.4$ & $184.1 \pm 260.0$ & n.s. ${ }^{\#}$ \\
\hline & Range & $16-390$ & $26-1200$ & \\
\hline \multirow[t]{2}{*}{$\begin{array}{l}\text { JC pollen- } \\
\text { specific IgE }\end{array}$} & Mean \pm SD & $16.4 \pm 13.2$ & $24.9 \pm 26.9$ & n.s. ${ }^{\#}$ \\
\hline & Range & $3.7-50.9$ & $4.6-100$ & \\
\hline \multirow[t]{2}{*}{$\begin{array}{l}\text { JC pollen- } \\
\text { specific } \\
\text { IgG4 }\end{array}$} & Mean $\pm S D$ & $0.28 \pm 0.39$ & $0.26 \pm 0.41$ & n.s. ${ }^{\#}$ \\
\hline & Range & $0-1.42$ & $0-1.34$ & \\
\hline
\end{tabular}

\#Student t-test.

IgE, immunoglobulin E; lgG4, immunoglobulin G4; SD, standard deviation; n.s., not significant.

tongue and to keep it in their mouth for 2 minutes before swallowing. The dosing schedule comprised an initial 2-week induction period in which the dose was titrated every day from 40 Japanese allergy units (JAU)/day to $2000 \mathrm{JAU} /$ day, followed by a maintenance period at a daily dose of $2000 \mathrm{JAU} /$ day, according to the protocol ${ }^{(9)}$. A $2000 \mathrm{JAU}$ dose of JC pollen extract contains 1.5-4.2 $\mu \mathrm{g}$ of the major allergen Cry j 1. Patients in the placebo group received inactive $50 \%$ glycerol in saline. All patients were allowed to take symptom-reducing drugs (fexofenadine hydrochloride $60 \mathrm{mg}$ tablet, tramazoline hydrochloride nasal solution $0.118 \%$, or ketotifen fumarate ophthalmic solution $0.05 \%$ ), as needed.

\section{Symptom evaluation}

Patients completed a pollinosis diary to record their nasal symptoms and use of symptom-reducing drugs during the 2011 and 2012 pollen seasons. Scores were obtained for nasal symptoms, sneezing, rhinorrhea, and nasal congestion using a 5-point scale (0: none, 1: mild, 2: moderate, 3 : severe, 4 : very severe) based on the Japanese guidelines for $\mathrm{AR}^{(13)}$. The use of each symptom relief medication was scored as 0 (not used) or 3 (used) and a total drug score was calculated (range $0-9^{(9)}$. The TNSMS (range 0-18) included the nasal symptom scores for sneezing, rhinorrhea, and nasal congestion and drug scores during the peak symptom period of the pollen season.

The peak symptom period was defined as the week with the worst symptoms, based on the sum of nasal symptom and medication scores for all patients, plus the weeks before and after 
this week in each pollen season. To avoid results being confounded by pollens from Japanese cypress (Chamaecyparis obtusa), which scatter after the cedar season, the peak symptom period was completed before 31 March. The average score for the peak pollen season was then calculated. Based on this definition, the evaluation periods were 7-27 March in 2011, and 19-31 March in 2012.

Assessment of symptoms and definition of responders Total JC pollen count at Chiba was 9199/cm² in 2011 and 3347/ $\mathrm{cm}^{2}$ in 2012. Considering the large number of dispersal of JC pollen in 2011 and a lower number in 2012, the criteria for symptom assessment were defined carefully. Based on the Japanese guidelines for $A R^{(13)}$, the severity of $A R$ in each patient was judged according to an average daily TNSMS during the peak symptom period (TNSMS $\leq 4$, mild symptoms; $\leq 7$, moderate; $\leq 10$, severe; $>10$, very severe). Based on severity, a "good" responder was defined as a patient who had at least moderate symptoms in 2011 and mild symptoms or an improvement in severity of two steps in 2012. A "poor" responder was defined as a patient who had at least moderate symptoms in 2011 and at least moderate symptoms and an improvement of one step or less in 2012. Patients with mild symptoms in 2011 were excluded because they could have had mild symptoms before SLIT. As a result, there were 13 good responders, 5 poor responders, and 2 exclusions in the SLIT group, and 7 good responders, 12 poor responders, and 1 exclusion in the placebo group.

\section{Blood samples}

Peripheral blood was obtained from each patient before initiation of dosing and before and at the end of the pollen seasons in 2011 and 2012. Peripheral blood mononuclear cells (PBMCs) and serum were isolated with Ficoll-Paque PLUS (GE healthcare Bio-Sciences, Uppsala, Sweden), and stored in a freezer at $-80^{\circ} \mathrm{C}$ until use.

\section{Measurement of serum immunoglobulins}

Serum levels of total $\lg \mathrm{E}$, JC pollen-specific $\lg \mathrm{E}$, and JC pollenspecific lgG4 were measured at Mitsubishi Chemical Medience (Tokyo, Japan).

\section{Measurement of JC pollen-specific IL- $10^{+} \mathrm{Foxp}^{+}$cells} The levels of JC pollen-specific IL- $10^{+} \mathrm{Foxp}^{+} \mathrm{CD} 4^{+} \mathrm{CD} 25^{+}$cells were analysed by flow cytometry ${ }^{(7)}$. Briefly, PBMCs were cultured with or without $10 \mu \mathrm{g} / \mathrm{mL}$ Cry j 1 and Cry j 2 for 3 days, followed by a culture with $10 \mathrm{ng} / \mathrm{mL}$ phorbol 12-myristate 13-acetate, $1 \mu \mathrm{M}$ ionomycin, and $2 \mu \mathrm{M}$ monensin for $6 \mathrm{~h}$. The PBMCs were stained with PE-Cy7-anti-CD4 antibody, APC-anti-IL10 antibody (BD Biosciences, San Diego, CA, USA), PE-anti-CD25, and FITCanti-Foxp3 (clone: $\mathrm{PCH} 101$ ) using a Foxp3 staining buffer set (eBioscience, San Diego, CA, USA). The number of IL-10+Foxp3 ${ }^{+}$
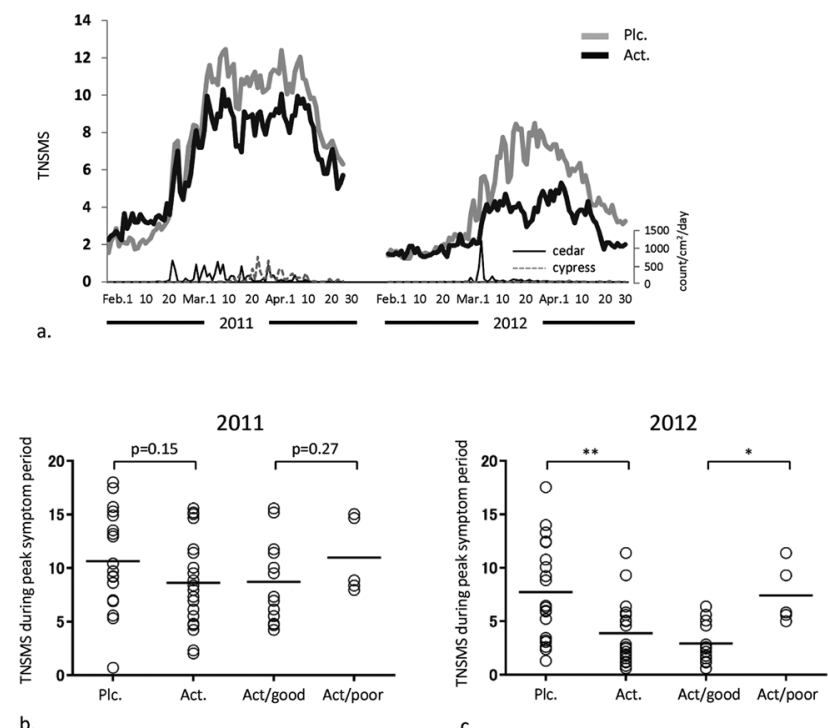

Figure 1. TNSMS in the pollen season in 2011 and in 2012.

(a) Mean total nasal symptom-medication scores (TNSMS) of patients during the pollen season and daily Japanese cedar and cypress pollen counts in 2011 and 2012 in Chiba, as measured by the Durham pollen sampler. Average daily TNSMS in the SLIT group (Act.; $N=20$ ), placebo group (Plc.; $\mathrm{N}=20$ ), good responders in the SLIT group (Act./good; $\mathrm{N}=13$ ) and poor responders in the SLIT group (Act/poor; $N=6$ ) are shown in the peak symptom period in 2011 (b) and in 2012 (c). Two-group comparisons were performed using an unpaired Student t-test. ${ }^{*} p<0.05$, ${ }^{* *} \mathrm{p}<0.01$.

cells was calculated in $10^{4}$ of $\mathrm{CD} 25^{+} \mathrm{CD} 4^{+}$cells.

Measurement of Th2 and Th1 type-cytokine-producing cells The numbers of JC pollen-specific IL-4, IL-5, IL-13 and interferon (IFN)- $\gamma$-producing cells were determined by enzyme-linked immunospot (ELISPOT) assay ${ }^{(7)}$. Briefly, a 96-well filter plate (Millipore, Billerica, MA, USA) was coated with monoclonal antibody to human IL-4, IL-5, IL-13, or IFN- $\gamma$ (Mabtech AB, Nacka Strand, Sweden). The plate was pre-incubated with AIM-V medium at $37^{\circ} \mathrm{C}$ for $1 \mathrm{~h}$. The medium was discarded, and PBMCs $\left(3 \times 10^{5}\right.$ cells/well) were then cultured with fresh medium alone or with $10 \mu \mathrm{g} / \mathrm{mL}$ Cry j 1 and Cry j 2 for $17 \mathrm{~h}$ at $37^{\circ} \mathrm{C}$ in AIM-V medium containing 5\% human AB serum (Sigma-Aldrich, St. Louis, MO, USA). The plates were then incubated with a biotinylated monoclonal antibody to human IL-4, IL-5, IL-13, or IFN- $\gamma$ for $2 \mathrm{~h}$, and then with streptavidin-conjugated alkaline phosphatase for $1 \mathrm{~h}$ at room temperature. After washing with $\mathrm{PBS}$, the plates were incubated with BCIP/NBT-plus (Mabtech AB) for 5 min at $37^{\circ} \mathrm{C}$, and the numbers of spots were counted with ImmunoScan ${ }^{\mathrm{TM}}$ (Cellular Technology Limited, OH, USA).

\section{Statistical analysis}

The Mann-Whitney $U$ test was performed to compare symptom- 

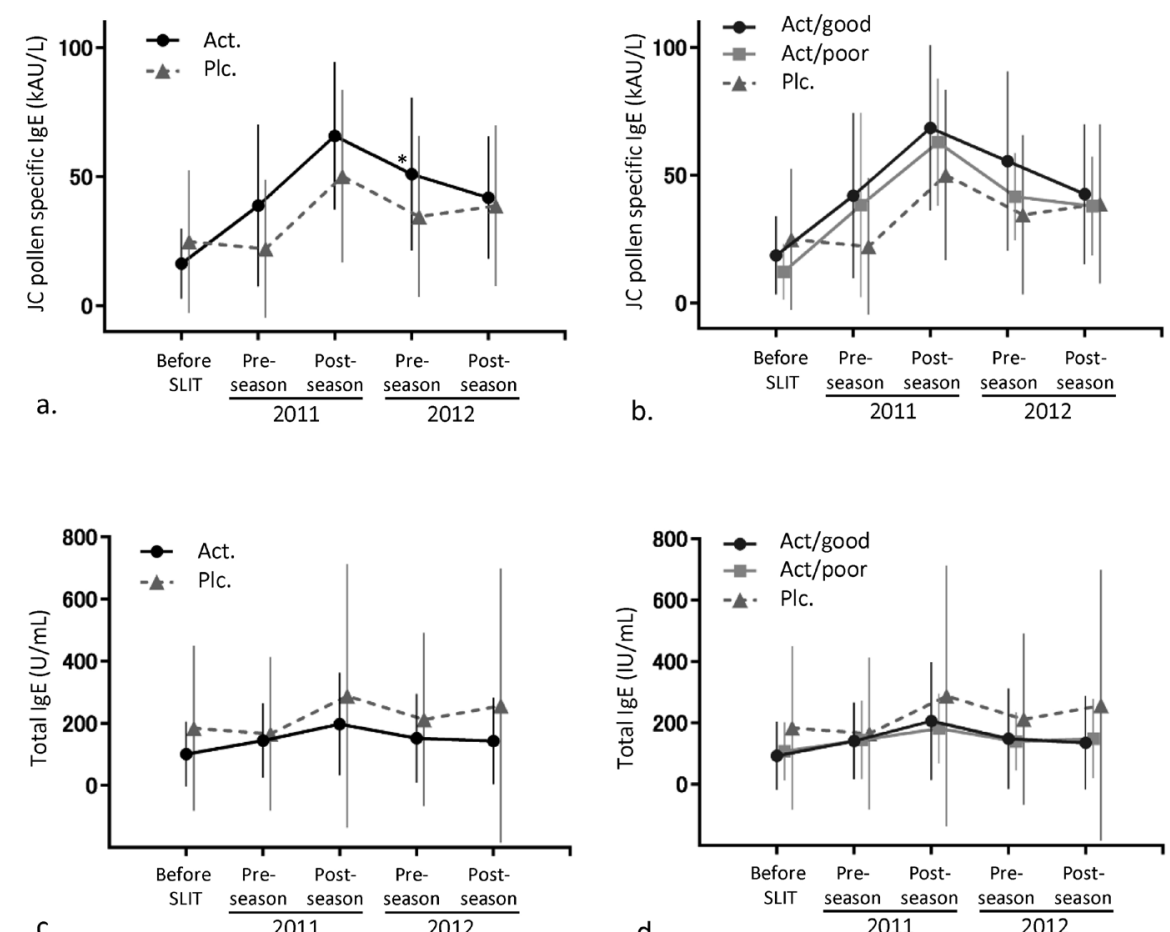

C.

d.
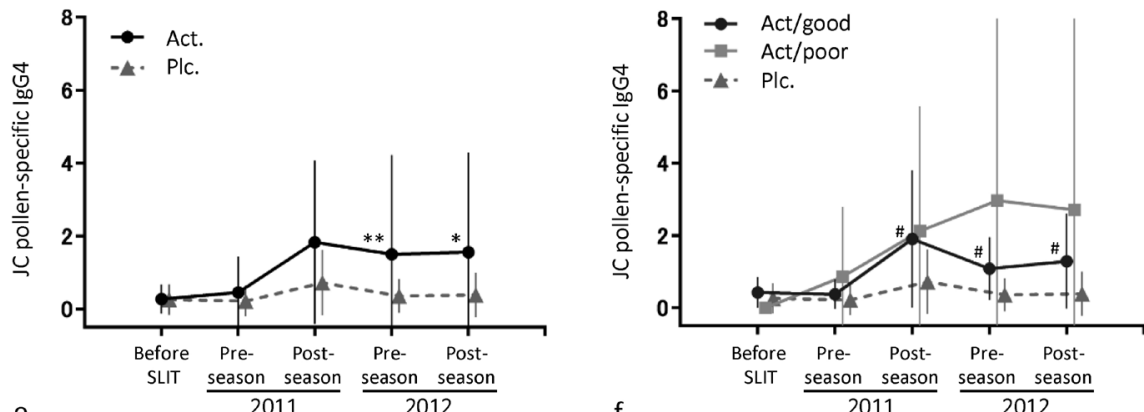

e.
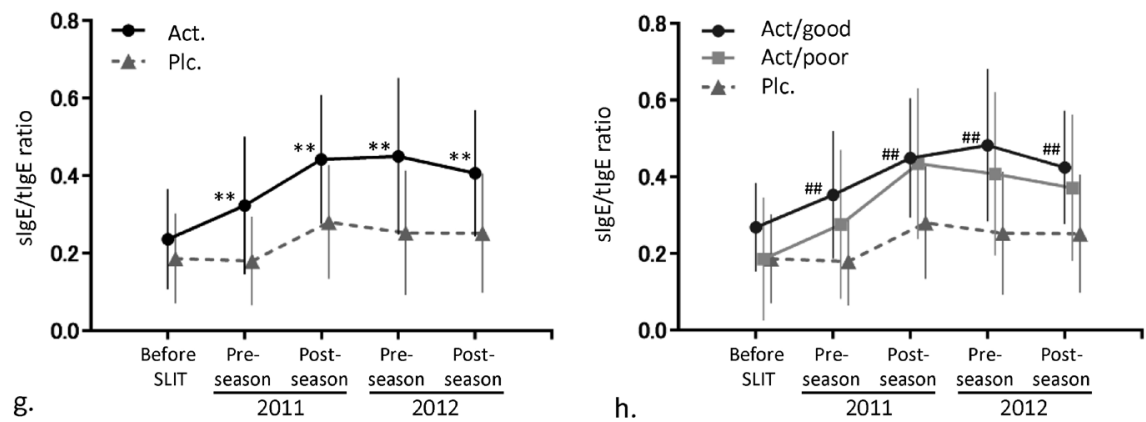

Figure 2. Serum immunoglobulins and clinical efficacy of SLIT. Serum levels of Japanese cedar (JC) pollen-specific lgE in the SLIT group (Act.) and in the placebo group (PIC.) (a), and in good responders in the SLIT group (Act/good) and poor responders in the SLIT group (Act/poor) (b). Serum levels of total IgE in the SLIT group (Act.) and placebo group (PIc.) (c), and in good responders (Act/good) and poor responders (Act/poor) in the SLIT group (d). Serum levels of JC pollen-specific IgG4 in the SLIT (Act.) and placebo group (PIc.) (e), and in good responders (Act/good) and poor responders (Act/poor) in the SLIT group (f). Specific IgE/total lgE (slgE/tlgE) ratios in the SLIT group (Act.) and placebo group (Plc.) (g), and in good responders (Act/good) and poor responders (Act/poor) in the SLIT group (h). Statistical analysis was performed using Mann-Whitney U test. ${ }^{*} p<0.05$, ${ }^{* *} p<0.01$ SLIT group vs. placebo group. \#p<0.05, \#\#p<0.01 SLIT good responder vs. placebo group. 


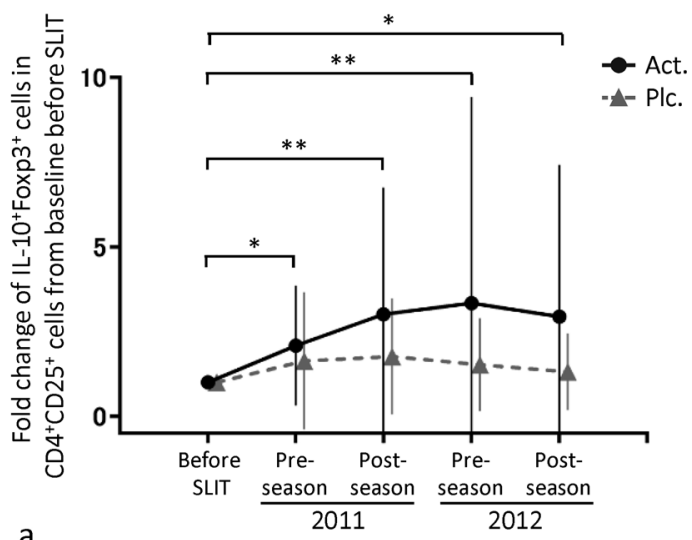

a.

Figure 3. Fold change of IL-10+Foxp3 $3^{+}$cells $/ \mathrm{CD} 4{ }^{+} \mathrm{CD} 25^{+}$cells. Fold change of $\mathrm{IL}-10^{+} \mathrm{Foxp} 3^{+}$cells $/ \mathrm{CD} 4{ }^{+} \mathrm{CD} 25^{+}$cells at each time point from baseline in the SLIT group (Act.) and the placebo group (PIC.) (a), and in good responders (Act/good) and poor responders in the SLIT group (Act/poor), and good responders (Plc/good) and poor responders (PIc/poor) in the placebo group (b). Statistical analysis was performed using the Wilcoxon signed-rank test. ${ }^{*} p<0.05,{ }^{* *} p<0.01$.

medication scores, and levels of immunoglobulins and cytokineproducing cells between groups. The Wilcoxon signed rank test was used for paired comparisons of the levels of IL- $10^{+} \mathrm{Foxp} 3^{+}$ cells and cytokine-producing $T$ cells before and after SLIT. Values of $p<0.05$ were considered statistically significant.

\section{Results}

\section{Clinical efficacy of SLIT in subgroups}

Data from symptom diaries were collected from all 40 patients, and the clinical efficacy of SLIT was analysed for the 2011 and 2012 peak symptom periods. The mean TNSMS was lower in the SLIT group compared with the placebo group in 2011 (Figure $1 \mathrm{a})$, but the difference was not significant during the peak symptom period (SLIT vs. placebo: 8.6 vs.10.6; $p=0.15$ ) (Figure 1b). The mean TNSMS was lower in the SLIT group compared with the placebo group from the beginning of the pollen season in 2012 (Figure 1a), and the difference was significant for the peak symptom period (SLIT vs. placebo: 3.9 vs. 7.7; $p<0.01$ ) (Figure 1c).

\section{Serum immunoglobulins and clinical efficacy of SLIT}

Serum levels of JC pollen-specific IgE in the SLIT group were comparable to those of the placebo group before treatment, but started to increase in the SLIT group at an early point after initiation of treatment. Although the level of JC pollen-specific IgE in the SLIT group was significantly higher than that in the placebo group in 2012 pre-pollen season, the IgE level was decreased post-pollen season in 2012 (Figure 2a). The specific IgE level in the good responders in the SLIT group was shifted in the same way to that for the poor responders in the SLIT group. There was no significant difference between the two groups (Figure 2b). There was no significant difference in serum total lgE levels between the SLIT group and the placebo group (Figure 2c), or between the good responders and poor responders in the SLIT group from pretreatment to the 2012 post-pollen season (Figure 2d).

Serum levels of JC pollen-specific IgG4 in the SLIT group were elevated in the post-pollen season in 2011, and there were significant differences in levels in the placebo group in the pre- and post-pollen seasons in 2012 (Figure 2e). The specific lgG4 level in the poor responders in the SLIT group was elevated in the same way as that in the good responders in the SLIT group in the post-pollen season in 2011. There was no significant difference between the two groups (Figure 2f).

The ratio of JC pollen-specific $\lg E$ to total $\lg \mathrm{E}$ (slgE/tlgE ratio) in the SLIT group was significantly higher than in the placebo group after the initiation of treatment (Figure $2 \mathrm{~g}$ ). However, there was no significant difference between the good responders and the poor responders in the SLIT group (Figure $2 \mathrm{~h}$ ). Serum levels of slgE, $\operatorname{tg} \mathrm{E}$, slgG4 and the ratio of slgE/tlgE changed to a similar extent in good and poor responders in the placebo group, and there was no significant difference at any time point (data not shown).

Association between Cry j-specific IL- $10^{+} \mathrm{Foxp} 3^{+}$cells and the efficacy of SLIT

The fold change of Cry j-specific IL- $10^{+} \mathrm{Foxp}^{+}$cells in $\mathrm{CD} 4^{+} \mathrm{CD} 25^{+}$ cells from baseline before SLIT was significantly increased in the SLIT group from the 2011 pre-pollen season, and continued to increase in 2012 (Figure 3a). The levels of the cells were significantly increased from baseline in the good responders in the SLIT group, but not in poor responders in the SLIT group (Figure $3 b)$. No significant difference in the levels of Cry j-specific IL-10+ Foxp $3^{+}$cells was observed between the good responders in the SLIT group and the poor responders in the SLIT group or the 

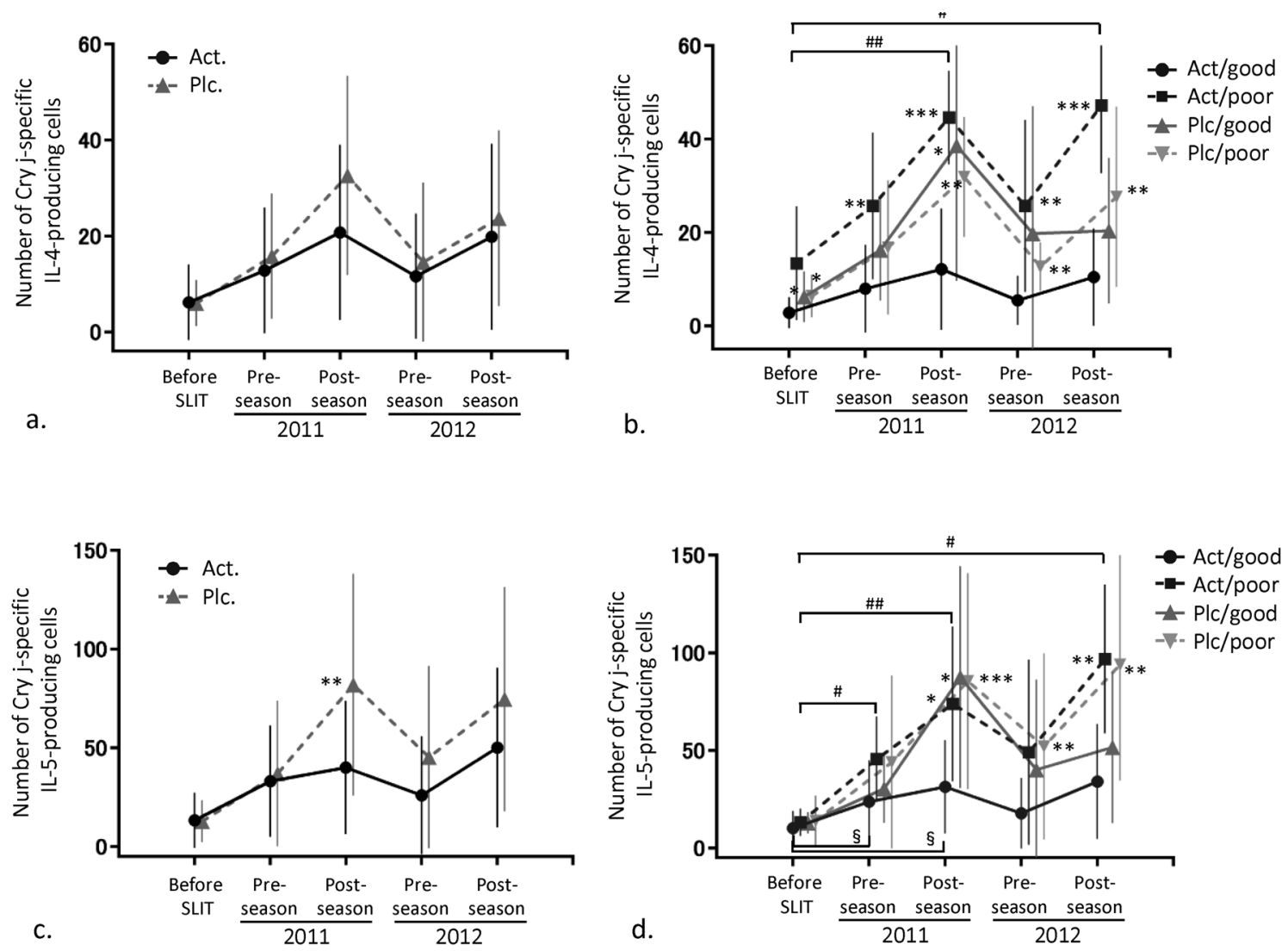

Figure 4. The number of Cry j-specific cytokine-producing cells. The number of Cry j-specific cytokine-producing cells in the SLIT group (Act.) and in the placebo group (PIc.) (IL-4: a, IL-5: c), and in good responders (Act/good) and poor responders (Act/poor) in the SLIT group, and in good responders (Plc/good) and poor responders (PIc/poor) in the placebo group (IL-4: b, IL-5: d). Statistical analysis was performed using Mann-Whitney U test. ${ }^{*} p<0.05,{ }^{* *} p<0.01,{ }^{* * *} p<0.001$, Act/poor, Plc/good or Plc/poor vs. Act/good. \#p<0.05,\#\#<0.01 in Act/poor. \$p $<0.05$ in Act/good.

good and poor responder subgroups in the placebo group at any time point. When the cutoff value for good clinical response to SLIT was defined as 3.5-fold that of IL- $10^{+} \mathrm{Foxp}^{+}$cells in this setting, sensitivity and specificity were $38.5 \%$ and $80.0 \%$, respectively, for the post-pollen season in 2011, and $35.7 \%$ and $100 \%$, respectively, in 2012.

Association between JC pollen-specific cytokine-producing cells and the efficacy of SLIT

The number of JC pollen-specific IL-4-producing cells in the placebo group was elevated in 2011 post-pollen season, but there was no significant difference between the SLIT and placebo groups (Figure 4a). However, the number of IL-4-producing cells in the good responders in the SLIT group was significantly lower than that in the poor responders in the SLIT group, and the good responders and poor responders in the placebo group ( $\mathrm{Fi}-$ gure $4 b$ ). The differences in the number of JC pollen-specific IL4-producing cells from before treatment to post-pollen season in 2011 and 2012 were significantly less in the good responders in the SLIT group than in the poor responders in the SLIT group $(p<0.05)$ (Figure 5a, 5b). When the cut-off value for good clinical response to SLIT was defined as 30 spots of IL-4-producing cells in this setting, sensitivity and specificity were $92.3 \%$ and $100 \%$, respectively, for the post-pollen season in 2011, and 100\% and $100 \%$, respectively, in 2012.

Similarly, the number of JC pollen-specific IL-5-producing cells in the SLIT group was significantly lower than in the placebo group at the 2011 post-pollen season (Figure 4c). The number of IL5 -producing cells in the good responders in the SLIT group was significantly lower than in the poor responders in the SLIT group and the good responders and poor responders in the placebo group at the post-pollen season (Figure $4 \mathrm{~d}$ ). The differences in the number of JC pollen-specific IL-5-producing cells from pretreatment to post-pollen season in 2011 and 2012 in the good responders in the SLIT group were significantly less than in the poor responders in the SLIT group $(p<0.05, p<0.01)$ (Figure $5 c$, $5 d$ ). When the cut-off value for good clinical response to SLIT was defined as 50 spots of IL-5-producing cells in this setting, sensitivity and specificity were $69.2 \%$ and $80.0 \%$, respectively, at post-pollen season in 2011 , and $76.9 \%$ and $100 \%$, respectively, 


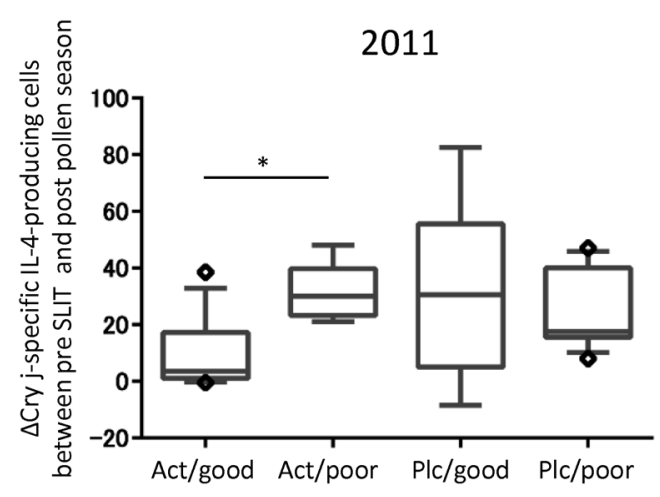

a.

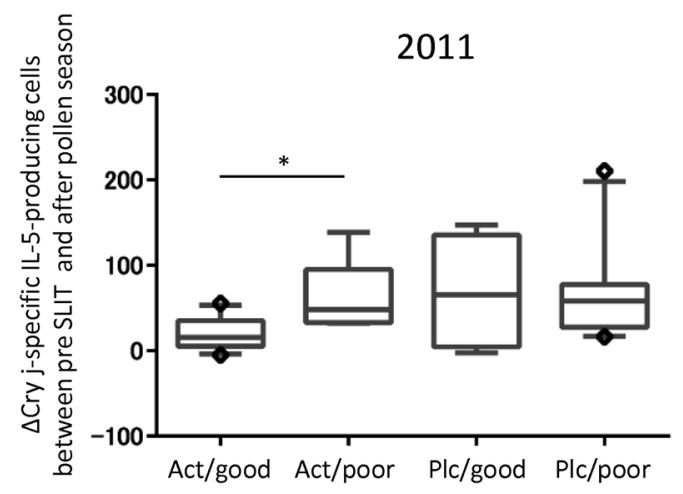

c.

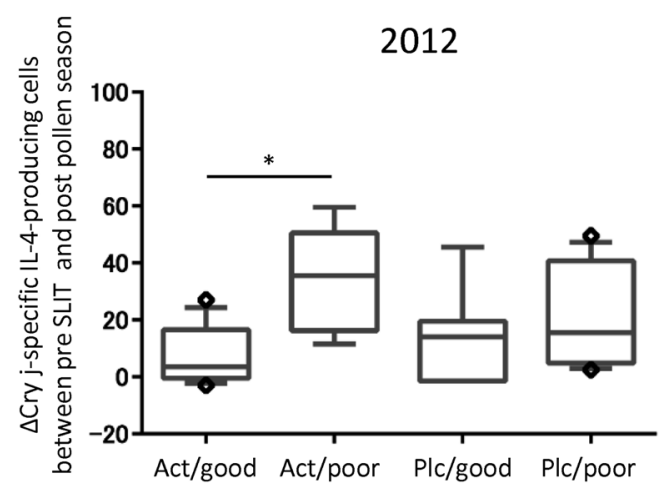

b.

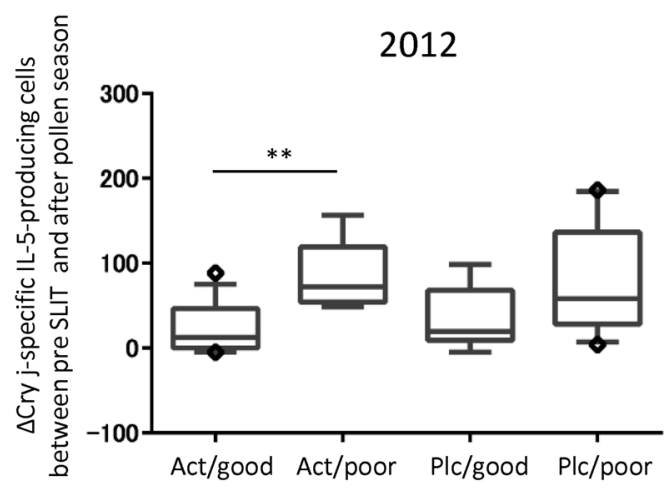

d.

Figure 5. Difference in the number of Cry j-specific IL4-producing cells between before SLIT and after pollen season. Difference in the number of Cry j-specific IL4-producing cells between before SLIT and after pollen season in 2011 and 2012 in the SLIT group (Act.) and in the placebo group (Plc.) (a), and in good responders (Act/good) and poor responders (Act/poor) in the SLIT group, and good responders (PIc/good) and poor responders (Plc/ poor) in the placebo group (b). Difference in the number of Cry j-specific IL5-producing cells in the SLIT group (Act.) and the placebo group (PIc.) (c), and in good responders (Act/good) and poor responders (Act/poor) in the SLIT group, and in good responders (Plc/good) and poor responders (PIc/ poor) in the placebo group (d). Statistical analysis was performed using the Mann-Whitney $\mathrm{U}$ test. ${ }^{*} p<0.05,{ }^{* *} p<0.01$.

in 2012. When therapeutic efficacy in 20 patients in the SLIT group was judged with the cut-off values of IL-4- and IL-5-producing cells in 2011, 14 patients were judged to be effective and 6 patients to be non-effective.

There were no significant differences in the number of IL-13or IFN- $\gamma$-producing cells between SLIT and placebo groups or between good responders and poor responders in the SLIT group at any time point (data not shown). There were no significant differences in the change in the number of IL-13- or IFN- $\gamma$-producing cells from pre-treatment to post-pollen season at each time point (data not shown).

\section{Discussion}

Although randomized, double-blind, placebo-controlled studies have examined the therapeutic efficacy of SLIT in patients with $A R$, and have a high level of evidence, the evaluation of therapeutic efficacy in pollinosis is difficult because scattering pollen amounts and climatic conditions vary each year, and placebo effects might influence the results for the treatment of AR. It was difficult to assess the therapeutic efficacy of treatment at the individual patient level.

In this study, in a subset of 40 patients who were enrolled in a large phase III trial of SLIT for JC pollinosis, the difference between the SLIT and placebo groups in mean TNSMS during the peak symptom period increased from the first pollen season to the second season, and nasal symptoms were significantly improved in the SLIT group despite the smaller amount of pollen dispersal in the 2012 pollen season. Symptoms were improved in the SLIT group as a whole during the two pollen seasons. However, there were also patients with only mild symptoms in the placebo group, as was the case in previous studies ${ }^{(7)}$. In our phase-III trial of SLIT for JC pollinosis, 39.4\% (95/241) of patients in the placebo group had only mild symptoms ( $<4$ points of TNSMS) during peak dispersal of pollen in the second season 
compared with $61.0 \%$ (147/241) of patients in the SLIT group ${ }^{(9)}$. In this analysis with 40 patients in our institute, 11 of 20 cases in the SLIT group and 5 of 20 subjects in the placebo group had mild symptoms during peak dispersal of pollen in the second season. The placebo effect should have influenced the SLIT group significantly, but making a distinction between the actual responder with non-responder from the results was difficult. To examine the biomarkers reflecting the efficacy of SLIT, patients were divided into subgroups according to the course of the nasal symptoms. Patients with mild symptoms in the first pollen season were excluded, and patients with mild symptoms and the patients whose symptoms improved two grades or more in the second season were defined as good responders, and the patients with moderate or more severe symptoms and those whose symptoms improved one grade or less in the second pollen season were defined as poor responders. By these criteria, the patients who had mild symptoms in the first year were excluded and the degree of improvement in each patient was taken into account.

In previous studies of SLIT, it has been suggested that SLIT induced specific IgG4 production and attenuated seasonal increase of Th2 cells, and increased the number of IL-10-producing cells and Treg cells, and that these markers are involved in the therapeutic mechanism of action ${ }^{(7,12,14)}$. Some recent studies have examined immunological changes in SLIT, but many of them were not conducted as placebo-controlled studies. In this study, we examined the association between the therapeutic efficacy based on the above-described assessment criteria and candidate biomarkers in 40 patients who took part in a phase III, randomized, double-blind, placebo-controlled trial of SLIT for JC pollinosis.

We examined the association between the levels of immunoglobulins and the efficacy of SLIT. An early increase and later decrease in specific IgE and an early and continuous increase in IgG4 have been reported during SLIT ${ }^{(12)}$. In particular, it has been suggested that specific lgG4 is involved in the mechanism of SLIT and works as a blocking antibody ${ }^{(11,16-18)}$. In this study, the level of serum JC pollen-specific IgE was elevated from an early point after initiation of SLIT, but decreased after the second pollen season. Because this behavior in the good responders in the SLIT group was comparable to that in the poor responders in the SLIT group, it was suggested that this elevation was affected by the administration of allergen and that it was not involved in the therapeutic efficacy of SLIT. There was no significant difference in serum total IgE levels between the SLIT group and the placebo group or between the good responders and the poor responders in the SLIT group, suggesting there was no association with efficacy. An elevation of the level of specific IgG4 was detected after the first pollen season in the SLIT group, and it was significant in the second year. However, the level of specific lgG4 in poor responders in the SLIT group was elevated as much as that in good responders in the SLIT group, and there was no significant difference between the two groups. The level of specific lgG4 in good responders (but not in poor responders) was significantly higher than that in the placebo group, but the wide distribution in poor responders might have influenced the results. It was suggested that specific lgG4 production was induced by the administration of allergen and the natural exposure to JC pollen, and that there was no association with the efficacy of SLIT.

In this study, the slgE/tlgE ratio increased from an early point after initiation of SLIT and was maintained at a significantly increased level during the treatment period. However, no significant difference was observed between good responders and poor responders in the SLIT group, suggesting there was no association with the efficacy of SLIT. In our previous study, we reported a possible association between the slgE/tlgE ratio before treatment and symptoms in the peak pollen season after SLIT ${ }^{(7)}$. However, it was not found to be a predictive marker for therapeutic efficacy in this study.

Th2 cytokine-producing cells in response to specific antigens are considered to be specific Th2 cells, and were another candidate marker examined in this study. Only the good responders in the SLIT group had significant attenuation of an increase of IL-4- and IL-5-producing Th2 cells in response to pollen dispersal from the first year after initiation of SLIT. Despite the small size of this study, the suppression of seasonal increases in specific IL-4- and IL-5-producing Th2 cells by SLIT was validated. This suggests that the measurement of these cells could be used as monitoring and predictive biomarkers for SLIT and could distinguish between the good responders and poor responders. In the ELISPOT assay, 30 spots of IL-4-producing cells and 50 spots of IL-5-producing cells at post-pollen season were suggested as cut-off values to determine the efficacy of SLIT. Furthermore, if the therapeutic efficacy in all 20 patients in the SLIT group was judged with cut-off values, 14 patients were judged to be effective and 6 patients to be non-effective in the first year. Two of the 6 patients judged to be non-effective were originally defined as good responders by the assessment based on the subjective symptom. Subjective improvement without the suppressed response of Th2 cells against pollen allergen in the SLIT group may not have been associated with the effects of SLIT. Therapeutic efficacy with measurement of Th2 cytokineproducing cells was judged by suppression of their seasonal increase, so judging efficacy in a year with a small amount of pollen dispersal would be difficult. In contrast, JC pollen-specific IL-10+Foxp $3^{+}$cells were increased continuously after initiation of SLIT in good responders, so they would be better markers in a year with a small amount of pollen dispersal. However, the number of patients with increased numbers of IL- $10^{+}$Foxp $3^{+}$cells was small in size compared with the number of patients with suppressed Th2 cytokine-producing cells in good responders in 
the SLIT group. The sensitivity was low but the specificity was high, so subjects with increased numbers of IL- $10^{+}$Foxp $3^{+}$cells in the second year might have been measured as part of the good responders. In the present situation, measuring $\mathrm{IL}-10^{+} \mathrm{Foxp}^{+}$ cells to judge therapeutic efficacy as an aid to measurement of Th2 cytokine-producing cells may be a better option. Carrying out these methods in the outpatient clinic can be difficult because they require special experimental facilities for measurement. Detection of pollen-specific Th2 cells using flow cytometry directly would be more convenient but is difficult because the antigen-tetramer method cannot cover detection of specific Th2 cells in all patients with JC pollinosis.

In the present study, antihistamine and decongestants were allowed to be used for rescue. These drugs can suppress activation of T cells ${ }^{(19-21)}$, but the number of medications was reduced in patients with mild symptoms. The use of antihistamines and decongestants did not affect the results appreciably (data not shown).

Allergen-specific IL- $10^{+}$Foxp $3^{+}$cells in $\mathrm{CD} 25^{+} \mathrm{CD} 4^{+}$cells were examined as a candidate biomarker in this study. These cells are considered to be a part of Foxp $3^{+}$Treg cells which produce inhibitory cytokine IL-10 in response to allergen stimulation. In this study, although no significant difference of IL- $10^{+} \mathrm{Foxp}^{+}$ cells was demonstrated between the good responders and poor responders in the SLIT group, IL- $10^{+} \mathrm{Foxp}^{+}$cells were significantly increased from an early time point after initiation of SLIT, and tended to increase further during the treatment period, suggesting that peripheral IL- $10^{+} \mathrm{Foxp} 3^{+}$Treg cells were associated with the therapeutic efficacy of SLIT. Increased Treg cells were reported after SLIT and suggested involvement in a mechanism of specific Th2 cell suppression ${ }^{(12,22)}$. However, in this study, no significant correlation between specific IL-10+Foxp3 ${ }^{+}$ cells and specific IL-4 or IL-5-producing cells was detected at any time point (data not shown). It is not fully understood to what extent the different factors, including Treg cells, contribute to the development of allergen tolerance by SLIT, and the effects of specific IL- $10^{+}$Foxp $3^{+}$cells on specific Th2 cells remains unclear. Because of the difference in total pollen count in each year, the differences in symptoms in each patient, and the involvement of placebo effects, it is difficult to evaluate therapeutic efficacy objectively at the individual patient level based on symptom or severity scores. SLIT can change the natural course of allergic rhinitis and is expected to elicit long-term therapeutic effects. SLIT is not a continuing treatment like other drug treatments, so a placebo effect by SLIT would not continue and disappear after treatment end. Exclusion of placebo effects using subjective assessments is generally difficult. Therefore, judgement of the therapeutic efficacy of SLIT based on objective assessments is of considerable importance to exclude placebo effects and to achieve the long-term effects of SLIT. In addition, although allergen immunotherapy requires a long time to accomplish treatment, a proportion of patients had no improvement in symptoms. Therefore, therapeutic efficacy can be predicted at an early time point, the burden would be reduced. If objective data showing good therapeutic efficacy can be shown to patients, they could be motivated to continue treatment, for which a high dropout rate has been reported ${ }^{(23)}$.

Antigen-specific IL-10+Foxp3+ cells and IL-4- or IL-5-producing $T$ cells are expected to be useful for monitoring and predicting the efficacy of SLIT. However, further validation studies in clinical practice are needed.

\section{Conclusion}

The association between biomarkers and therapeutic efficacy was examined in 40 patients from a phase III, randomized, double-blind, placebo-controlled study of SLIT. Increased IL-10+Foxp $3^{+}$cells and suppression of IL-4- and IL-5-producing cells were found to correlate with the efficacy of SLIT, which was assessed based on the clinical course of nasal symptoms after treatment. These markers could be useful for monitoring and predicting the efficacy of SLIT, and could discriminate between good responders and poor responders to SLIT.

\section{Acknowledgements}

We sincerely thank Ayako Inamine for supporting the experiments, Koichi Nakano (Nakano ENT Clinic) and Minako Hisamitsu (Chiba Kaihin Municipal Hospital) for conducting the clinical study, and Osamu Ohara (Kazusa DNA research institute) and Ken Nonaka (DNA chip Research Inc.) for providing the statistical analysis and helpful comments. This work was supported by Torii Pharmaceuticals and in part by a grant from the Japanese Ministry of Health, Labor and Welfare.

\section{Authorship contribution}

Daiju Sakurai, Syuji Yonekura, Shinya Kaneko, Yoshitaka Okamoto designed and developed the protocol for the study. Daiju Sakurai, Syuji Yonekura, Toshioki Sakurai conducted the clinical study, assisted by Yuki Morimoto, Yukisoshi Mita, Tomoyuki Arai, Satoshi Suzuki, Yusuke Okuma, Tomohisa linuma. Daiju Sakurai performed the analysis of results and wrote the manuscript. Yoshitaka Okamoto supervised the study and reviewed the manuscript.

\section{Conflict of interest}

Yoshitaka Okamoto has received payments for consultancies from Torii Pharmaceuticals, Shionogi, and MSD. Tomohisa linuma belongs department of Advanced Allergology of the Airway which is a endow course by Torii Pharmaceuticals. The remaining authors have no competing interests to declare. 


\section{References}

1. Di Bona D, Plaia A, Scafidi V, Leto-Barone MS, Di Lorenzo G. Efficacy of sublingual immunotherapy with grass allergens for seasonal allergic rhinitis: A systemic review and meta-analysis. J Allergy Clin Immunol 2010; 126:558-566.

2. Radulovic S, Calderon MA, Wilson D, Durham S. Sublingual immunotherapy for allergic rhinitis. Cochrane Database Syst Rev 2010; CD002893.

3. Radulovic S, Wilson D, Calderon M, Durham S. Systematic review of sublingual immunnotherapy (SLIT). Allergy 2011; 66:740-752.

4. Lin SY, Erekosima N, Kim JM, et al. Sublingual immunotherapy for the treatment of allergic rhinoconjunctivitis and asthma. JAMA 2013; 309:1278-1288.

5. Horiguchi S, Okamoto Y, Yonekura S, et al. A randomized controlled trial of sublingual immunotherapy for Japanese cedar pollinosis. Int Arch Allergy Immunol 2008; 146:76-84.

6. Fujimura T, Yonekura S, Taniguchi Y, et al. The induced regulatory $T$ cell level, defined as the proportion of $\mathrm{IL}-10(+)$ Foxp3(+) cells among CD25(+)CD4(+) leukocytes, is a potential therapeutic biomarker for sublingual immunotherapy: a preliminary report Int Arch Allergy Immunol 2010; 153:378387.

7. Fujimura T, Yonekura S, Horiguchi S, et al. Increase of regulatory $T$ cells and the ratio of specific IgE to total IgE are candidates for response monitoring or prognostic biomarkers in 2-year sublingual immunotherapy (SLIT) for Japanese cedar pollinosis. Clin Immunol 2011; 139:65-74.

8. Okubo K, Gotoh M, Fujieda S, et al. A randomized double-blind comparative study of sublingual immunotherapy for cedar pollinosis. Allergol Int 2008; 57:265-275.

9. Okamoto Y, Okubo K, Yonekura S, H et al. Efficacy and Safety of Sublingual Immunotherapy for Two Seasons in Patients with Japanese Cedar Pollinosis. Int Arch Allergy Immunol 2015; 166:177-188.

10. Piconi S, Trabattoni D, Rainone $V$, et al. Immunological effects of sublingual immunotherapy: clinical efficacy is associated with modulation of programmed cell death ligand 1, IL-10, and IgG4. J Immunol 2010; 185:7723-7730

11. Scadding GW, Shamji MH, Jacobson MR, et al. Sublingual grass pollen immunotherapy is associated with increases in sublingual Foxp3-expressing cells and elevated allergen-specific immunoglobulin G4, immunoglobulin A and serum inhibitory activity for immunoglobulin E-facilitated allergen binding to B cells. Clin Exp Allergy 2010; 40:598-606.

12. Akdis M, Akdis CA. Mechanisms of allergenspecific immunotherapy: multiple suppressor factors at work in immune tolerance to allergens. J Allergy Clin Immunol 2014; 133:621-631

13. Fujieda S, Kurono Y, Okubo K, et al Examination, diagnosis and classification for allergic rhinitis: Japanese guideline. Auris Nasus Larynx 2012; 39:553-556.

14. James LK, Shamji MH, Walker SM, et al. Long-term tolerance after allergen immunotherapy is accompanied by selective persistence of blocking antibodies. J Allergy Clin Immunol 2011; 127:509-516, e1-5.

15. Fujimura T, Okamoto $Y$, Taniguchi $M$. Therapeutic effects and biomarkers in sublingual immunotherapy: a review. J Allergy 2012; 381737

16. Wachholz PA, Durham SR. Mechanisms of immunotherapy: IgG revisited. Curr Opin Allergy Clin Immunol 2004; 4:313-318.

17. Flicker $S$, Valenta R. Renaissance of the blocking antibody concept in type I allergy. Int Arch Allergy Immunol 2003; 132:13-24.

18. Nouri-Aria KT, Wachholz PA, Francis JN, et al. Grass pollen immunotherapy induces mucosal and peripheral IL-10 responses and blocking IgG activity. J Immunol 2004;
172:3252-3259

19. Botturi K, Lacoeuille $Y$, Vervloet $D$ Magnan A. Histamine induces Th2 activation through the histamine receptor 1 in house dust mite rhinitic but not asthmatic patients. Clin Exp Allergy 2010; 40:755-62.

20. Okamoto T, Iwata S, Ohnuma K, Dang NH, Morimoto C. Histamine $\mathrm{H1}$-receptor antagonists with immunomodulating activities: potential use for modulating $T$ helper type 1 (Th1)/Th2 cytokine imbalance and inflammatory responses in allergic diseases. Clin Exp Immunol 2009; 157:27-34.

21. Fiebich BL, Collado JA, Stratz C, et al. Pseudoephedrine inhibits T-cell activation by targeting NF-KB, NFAT and AP-1 signaling pathways. Immunopharmacol Immunotoxicol 2012; 34:98-106.

22. John W, Steinke JW, Lawrence MG. T-cell biology in immunotherapy. Ann Allergy Asthma Immunol 2014; 112:195-199.

23. Antico A. Long-term adherence to sublingual therapy: literature review and suggestions for management strategies based on patients' needs and preferences. Clin Exp Allergy 2014; 44:1314-26.

\section{Yoshitaka Okamoto MD, PhD Department of Otolaryngology Head and Neck Surgery Graduate School of Medicine Chiba University \\ 1-8-1 Inohana, Chuo-ku Chiba 260-8670 \\ Japan}

Tel: +81-43-226-2137

Fax: $+81-43-227-3442$

E-mail: yokamoto@faculty.chiba-u.jp 